\title{
Nadeln für kürzere Geburten
}

In der Geburtshilfe sind komplementärmedizinische Methoden sehr gefragt; so ist z.B. die Akupunktur bei Hebammen und Gynäkologen etabliert. Bei werdenden Müttern geniessen z.B. Aromatherapie, Klassische Homöopathie, Phytotherapie und Akupunktur, die zur Geburtsvorbereitung und -erleichterung eingesetzt werden, eine hohe Akzeptanz. Insbesondere die Akupunktur hat sich etabliert. In der Schweiz haben knapp 30 niedergelassene Gynäkologinnen und Gynäkologen eine von der FMH anerkannte Weiterbildung in Akupunktur-TCM; zudem lassen sich immer mehr Hebammen in geburtsvorbereitender Akupunktur aus- und weiterbilden.

So hat sich die geburtsvorbereitende Akupunktur zum Teil auch im klinischen Umfeld etabliert, z.B. in der Universitätsklinik für Frauenheilkunde des Inselspitals Bern. Dort ist das Angebot ausschliesslich in

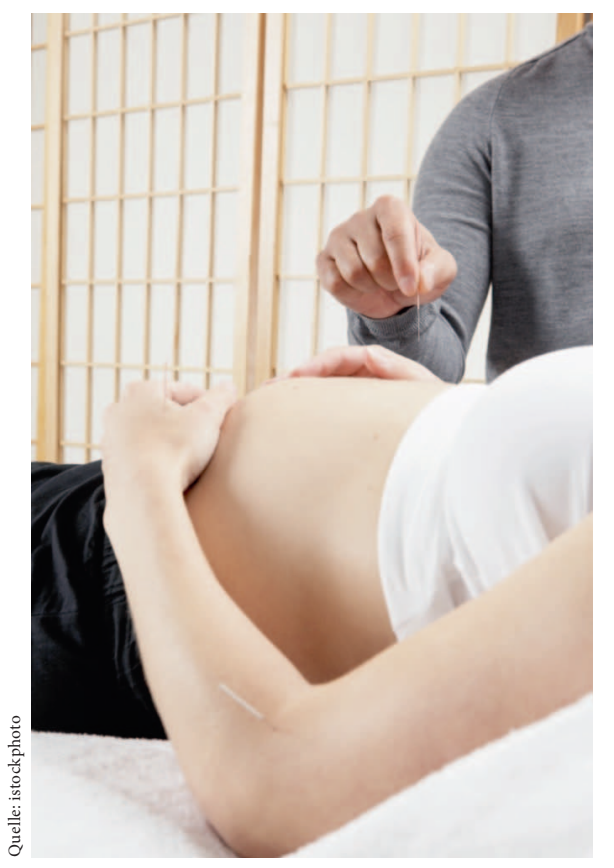

den Händen der Hebammen, die zu diesem Zweck die Kurse des ZhongInstituts (Konolfingen) besuchen. Das Konzept im Inselspital sieht wöchentliche Sprechstunden ab der 37. Schwangerschaftswoche bis zur Geburt vor, mit 3 Sitzungen à 4560 min. Die Kosten - 50 CHF pro Sitzung - müssen die Patientinnen selbst übernehmen, da es sich bei der geburtsvorbereitenden Akupunktur nicht um eine Pflichtleistung der Grundversicherung handelt.

Die klinische Forschung hat gezeigt, dass Akupunktur den Frauen zu leichteren und kürzeren Geburten verhilft. Die bekannteste Untersuchung ist diejenige von Ansgar Römer und seinen Mitarbeitenden von der Universitäts-Frauenklinik in Mannheim: Sie konnten Mitte der 1990erJahre in einer randomisierten Studie mit über 800 Schwangeren zeigen, dass sich die Geburtszeit um ca. 2 h verkürzte, wenn die Schwangeren ab der 36. Woche nach einem bestimmten Schema am Bein und am Fuss akupunktiert wurden («Mannheimer Schema»). Dabei war die Eröffnungsphase - die Zeit vom Einsetzen der Wehen bis zur vollständigen Eröffnung des Muttermundes - verkürzt, die Austreibungsphase hingegen unterschied sich nicht. TCM-Spezialistinnen und -Spezialisten erklären dies mit einer besseren «Reifung» des Muttermundes. «Die geburtsvorbereitende Akupunktur hilft bei der Reifung der Geburtswege und harmonisiert die haltenden Yin-Kräfte, indem sie 4 Wochen vor der Geburt das Fliessen von Qi fördert», hält Römer fest. Durch die Akupunktur würden auch Blockaden in anderen Meridianen des Körpers gelöst und das allgemeine Fliessen der Lebenskräfte gefördert.
Römer hat zu diesem Thema mehrere Bücher geschrieben $[1,2]$.

Ausser zur Geburtsvorbereitung hat sich die Akupunktur zur Unterstützung während der Geburt durchgesetzt. Die Hebammen setzen entspannende und schmerzlindernde Akupunkturpunkte, die sie zur Verbesserung der Wirksamkeit stimulieren. Erfahrungsgemäss kann dieses Prozedere einen grossen Teil der Geburtsschmerzen lindern, und es hilft der werdenden Mutter, sich zu entspannen. «Der Vorteil ist, dass die Akupunktur keine Nebenwirkungen auf das Kind hat», sagt die TCMSpezialistin Kirsten Stähler van Amerongen, stellvertretende Chefärztin Gynäkologie und Geburtshilfe des Spitalzentrums Biel.

Auch bei Beckenendlagen des Kindes im Mutterbauch kann die Akupunktur Abhilfe schaffen. «Hier können wir ca. ab der 34. Schwangerschaftswoche mit Moxibustion am kleinen Zeh häufig eine Wendung des Kindes in eine Schädellage erreichen», sagt der Gynäkologe Andreas Guggisberg (siehe auch Expertengespräch). Die Zigarre aus Beifusskraut wird dabei intervallartig in die Nähe eines Punktes am kleinen Zeh gehalten. Arbeiten aus China bescheinigen diesem einfachen Verfahren eine Erfolgsquote von 90\%. Diese Ergebnisse liessen sich in europäischen Untersuchungen allerdings nicht bestätigen. (rfi)

\section{Literatur}

1 Römer A: Akupunktur für Hebammen, Geburtshelfer und Gynäkologen. Ein Lehrbuch der Chinesischen Medizin. Stuttgart, Hippokrates, 2008.

2 Römer A: Medical Acupuncture in Pregnancy. Stuttgart, Thieme, 2005. 